\title{
A GEOMETRIC SCHUR FUNCTOR
}

\author{
CARL MAUTNER
}

\begin{abstract}
We give geometric descriptions of the category $C_{k}(n, d)$ of rational polynomial representations of $G L_{n}$ over a field $k$ of degree $d$ for $d \leq n$, the Schur functor and Schur-Weyl duality. The descriptions and proofs use a modular version of Springer theory and relationships between the equivariant geometry of the affine Grassmannian and the nilpotent cone for the general linear groups. Motivated by this description, we propose generalizations for an arbitrary connected complex reductive group of the category $C_{k}(n, d)$ and the Schur functor.
\end{abstract}

2010 Mathematics Subject Classification. Primary 20G43, 14F05; Secondary 17B08.

Key words and phrases. modular Springer theory; Schur algebra; Schur functor; perverse sheaves; nilpotent cone; affine Grassmannian.

\section{INTRODUCTION}

1.1. In his 1901 thesis, Schur defined a functor from polynomial representations of $G L_{n}(\mathbb{C})$ of degree $d$ to representations of the symmetric group $\mathfrak{S}_{d}$, which is an equivalence of categories for $n \geq d$. Later it was observed by Green [Gre80] that Schur's functor is well defined over any field $k$, but is not an equivalence if the characteristic of $k$ is less than or equal to $d$. Green defines the Schur functor as follows. Consider the group $G L_{n}$ and let $C_{k}(n, d)$ denote the category of rational polynomial representations of $G L_{n}$ over $k$ of degree $d$. The $n$-dimensional standard representation $E$ of $G L_{n}$ is polynomial and homogeneous of degree one. Letting the symmetric group $\mathfrak{S}_{d}$ act on $E^{\otimes d}$ by permutations on the right defines a functor:

$$
\operatorname{Hom}\left(E^{\otimes d},-\right): C_{k}(n, d) \rightarrow k \mathfrak{S}_{d^{-}} \text {Mod. }
$$

The categories $C_{k}(n, d)$ and $k \mathfrak{S}_{d}$-Mod are much more complicated when char $k \leq$ $d$. The resulting categories are not semisimple and even basic facts, for example, the dimensions of the irreducible representations of the symmetric group, are not known in general. The Schur functor has been used as an effective tool to relate structure in the representation theory of the general linear groups to that of the symmetric groups and vice versa (e.g., [Don87, Don93, Kle95]).

1.2. In the first part of this paper, we relate the existence of the Schur functor to the geometry of certain singular spaces associated to $G L_{n}$. For $n \geq d$, we give a geometric interpretation of the category $C_{k}(n, d)$ and the Schur functor in terms of Springer theory for the nilpotent cone $\mathcal{N}_{d} \subset \mathfrak{g l}_{d}$. More precisely, we show:

Theorem 1.1. For any $n \geq d$, there is an equivalence of categories:

$$
\phi^{\bullet}: C_{k}(n, d) \stackrel{\sim}{\rightarrow} P_{G L_{d}}\left(\mathcal{N}_{d} ; k\right),
$$

where $P_{G L_{d}}\left(\mathcal{N}_{d} ; k\right)$ is the category of equivariant perverse sheaves with coefficients in $k$, which takes the representation $E^{\otimes d}$ to the Springer sheaf $\mathcal{S}$.

The author was supported by an NSF postdoctoral research fellowship. 
In Section 5, we use this result to provide a geometric proof of Carter-Lusztig's generalization of Schur-Weyl duality [CL74, Thm. 3.1].

Theorem 1.2. For any $d \leq n$, the morphism

$$
k \mathfrak{S}_{d} \rightarrow \operatorname{End}_{G L_{n}^{k}}\left(E^{\otimes d}\right)
$$

defined by permuting the tensor factors is an isomorphism.

Corollary 1.3. There is a commutative diagram of functors:

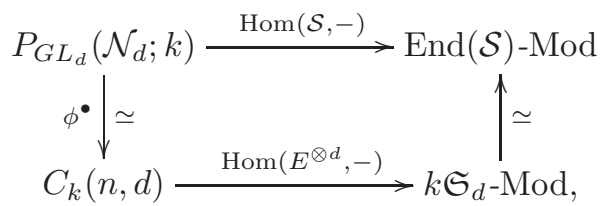

1.3. The proof of these statements is reached by crossing two bridges.

The first bridge is the geometric Satake equivalence (cf. Section 2.7), which relates the representation theory of a split reductive group $G$ over $k$ to a category of equivariant perverse sheaves on the affine Grassmannian for the complex reductive group $G_{\mathbb{C}}^{\vee}$ with dual root datum. In particular, it allows us to identify the category of polynomial representations $C_{k}(n, d)$ with equivariant perverse sheaves on a closed subvariety $\overline{\mathcal{G r}} \boldsymbol{r}^{\mathbf{d}}$ (cf. 2.6.1) of the affine Grassmannian $\mathcal{G} r_{G L_{n}}$.

The second bridge is a relationship between the nilpotent cone and the affine Grassmannian for the group $G L_{n}$. In the paper [Lus81], Lusztig introduced a map $\phi: \mathcal{N}_{d} \rightarrow \overline{\mathcal{G r}^{\mathrm{d}}}$. Using a map in the other direction defined on quotient stacks, we prove (Theorem 4.1) an equivalence of categories of equivariant perverse sheaves.

1.4. In the second part of the paper, we shift our focus to an arbitrary connected complex reductive group $G$. We observe that our reinterpretation of the Schur functor as a functor from the category of adjoint-equivariant perverse sheaves on the nilpotent cone to modules over endomorphisms of the Springer sheaf is welldefined for any reductive group.

Moreover, we give a reformulation in terms of the Fourier-Sato transform $\mathbb{T}$ on the Lie algebra $\mathfrak{g}$. Let $j_{\mathrm{rs}}: \mathfrak{g}_{\mathrm{rs}} \hookrightarrow \mathfrak{g}$ denote the open embedding of the regular semisimple locus and $i: \mathcal{N} \hookrightarrow \mathfrak{g}$ the closed embedding of the nilpotent cone. Consider the functor

$$
\mathcal{F}:=j_{\mathrm{rs}}^{*} \circ \mathbb{T} \circ i_{*}: P_{G}(\mathcal{N} ; k) \rightarrow P_{G}\left(\mathfrak{g}_{\mathrm{rs}} ; k\right),
$$

where $P_{G}(X ; k)$ denotes the category of $G$-equivariant perverse sheaves on a $G$ variety $X$. We show that $\mathcal{F}$ factors through $\operatorname{Loc}_{W}\left(\mathfrak{g}_{\mathrm{rs}}\right)$, the category of local systems on $\mathfrak{g}_{\mathrm{rs}}$ with Weyl group monodromy shifted by dim $\mathfrak{g}$ to be perverse, and identify $\mathcal{F}$ with the functor $\operatorname{Hom}(\mathcal{S},-)$.

We propose that $P_{G}(\mathcal{N} ; k)$ should be thought of as a generalization of the category $C_{k}(n, d)$ and $\mathcal{F}$ as the geometric Schur functor for the group $G$.

1.5. Related work. This paper is a revised version of part of the author's Ph.D. thesis [Mau10].

A generalization of some of the results in this paper has since been explored by Achar, Henderson and Riche. In [AH12] and then in [AHR12], they consider a split reductive group $G$ over $k$ and the category of 'small' representations of $G$ (analogous to a variant of $C_{k}(n, d)$ ). They show that the corresponding part of 
the affine Grassmannian for the dual group $G_{\mathbb{C}}^{\vee}$ contains an open locus that maps $G^{\vee}$-equivariantly to the nilpotent cone for $G^{\vee}$. They use this map to construct a functor $\Psi_{G}$ between categories of perverse sheaves on the affine Grassmannian and nilpotent cone. The main result of these papers is an equivalence between the composition of $\Psi_{G}$ with the geometric Schur functor and the composition of the Satake equivalence with taking the zero weight space together with its $W$-action.

Another closely-related picture arises in work of Achar and the author. Motivated by the equivalence between $C_{k}(n, d)$ and $P_{G L_{n}}(\mathcal{N} ; k)$, we propose [AM12] a geometric Ringel functor for the equivariant derived category $D_{G}(\mathcal{N} ; k)$. The definition is similar to that of the geometric Schur functor in this paper. In particular, we define a functor

$$
\mathcal{R}:=i^{*} \mathbb{T} i_{*}[\operatorname{dim} \mathcal{N}-\operatorname{dim} \mathfrak{g}]: D_{G}(\mathcal{N} ; k) \rightarrow D_{G}(\mathcal{N} ; k)
$$

and show that it is an autoequivalence.

We expect that the functors $\mathcal{F}$ and $\mathcal{R}$ will be useful tools in understanding the categories $P_{G}(\mathcal{N} ; k)$ and $D_{G}(\mathcal{N} ; k)$. Since this paper was submitted, work in this direction for $G=G L_{n}$ has appeared in [AHJR13], in which the results of Sections 6 and 7 are extended to define a stratification or iterated 'recollement' of the category $P_{G L_{n}}\left(\mathcal{N}_{d} ; k\right) \cong C_{k}(n, d)$.

1.6. Here is an outline of the paper. Section 2 contains a summary of the various ingredients that will be used in the paper. In Section 3, we study various maps between the nilpotent cone and affine Grassmannian and the relations they satisfy. We then use these relations in Section 4 to prove an equivalence of categories of perverse sheaves, with which, in Section 5, we deduce Carter-Lusztig's version of Schur-Weyl duality as a corollary. Sections 6 and 7 contain a proposal for a 'geometric Schur functor.'

1.7. Acknowledgments. This paper has been a long time coming and so the author had a number of years to benefit from useful conversations and deep insights from many people. He would like to thank in particular: David Ben-Zvi for continued support and advice, Daniel Juteau whose thesis was a source of inspiration for much of this paper, Pramod Achar for encouragement, Geordie Williamson for comments on a draft of the paper and an anonymous referee for a careful reading and many helpful comments. Thanks as well to Dennis Gaitsgory, Joel Kamnitzer, David Helm, David Nadler, Catharina Stroppel, Zhiwei Yun, and Xinwen Zhu.

\section{Dramatis Personae}

2.1. Notation. For a scheme $X$ defined over the complex numbers with an action of a reductive group $G$, we denote by $P_{G}(X ; k)$ (respectively $\left.D_{G}(X ; k)\right)$ the category of $G$-equivariant perverse sheaves (respectively the $G$-equivariant derived category) with coefficients in $k$ on $X$. This is equivalent to the category of perverse sheaves (respectively the constructible derived category) on the quotient stack $[X / G]$ (cf. [LO09, Rmk. 5.5]). For a locally closed and $G$-invariant subscheme $Y \subset X$ and a $G$-equivariant irreducible local system $\mathcal{L}$ on $Y$ shifted to be perverse, we denote by $\operatorname{IC}(Y, \mathcal{L}) \in P_{G}(X ; k)$ the simple perverse sheaf defined by the intersection cohomology complex on $Y$ with coefficients in $\mathcal{L}$. We denote the constant sheaf on $X$ by $\underline{k}_{X}$. 
We denote the general linear group $G L_{r}$ by $G_{r}$ and its Lie algebra by $\mathfrak{g}_{r}$. We consider the group $G_{r}$ over the field $k$. We fix the standard upper triangular Borel subgroup $B_{r}$ of $G_{r}$, with its unipotent radical $U_{r}$. Let $T_{r} \cong \mathbb{G}_{m}^{r}$ be the Cartan subgroup of diagonal matrices and $\mathfrak{h}_{r} \cong \mathbb{A}^{r}$ its Lie algebra, the standard Cartan subalgebra. The Weyl group $W_{r}=\mathfrak{S}_{r}$ acts on $\mathfrak{h}_{r}=\mathbb{A}^{r}$ by permuting its coordinates. We denote the weight lattice of $G_{r}$ by $\Lambda_{r}$ and identify it with $\mathbb{Z}^{r}$ using our identification of the Cartan subgroup. The set of dominant weights $\Lambda_{r}^{+}$are those $\lambda=\left(\lambda_{1}, \ldots, \lambda_{r}\right) \in \Lambda_{r}$ such that $\lambda_{i} \geq \lambda_{j}$ for all $i<j$.

For $n$ and $d$ positive integers, we denote the category of rational homogeneous polynomial representations of $G_{n}$ of degree $d$ by $C_{k}(n, d)$ (cf. [Jan03, A.3] for the definition). Let $\Lambda(n, d) \subset \Lambda_{n}$ be the set of weights $\left(\lambda_{1}, \lambda_{2}, \ldots, \lambda_{n}\right)$ such that $\sum_{i} \lambda_{i}=d$ and $\lambda_{i} \geq 0$ for all $1 \leq i \leq n$. As explained in [Jan03, Prop. A.3], if follows from work of Donkin [Don87] that the category $C_{k}(n, d)$ coincides with the full subcategory of those rational representations all of whose weights lie in the subset $\Lambda(n, d)$.

We now fix $n \geq d$. In what follows, we will give a geometric description of the category $C_{k}(n, d)$ that does not depend on $n$. It is well-known that for any $n>d$, there is an equivalence $C_{k}(n, d) \cong C_{k}(d, d)$ (see [Mar93, Thm 4.3.6]).

Remark 2.1. Many of the proofs and results of this paper hold when the field $k$ is replaced by any Noetherian commutative ring $\mathbf{k}$ of finite global dimension.

In this more general setting, $C_{\mathbf{k}}(n, d)$ should be interpreted as the full subcategory of rational representations $M$ of $G L_{n}$ over $\mathbf{k}$ such that the weight module $M_{\lambda}=0$ for all $\lambda \notin \Lambda(n, d)$. As mentioned above, when $\mathbf{k}$ is a field this is known to coincide with the category of polynomial representations of degree $d$.

With this interpretation of $C_{\mathbf{k}}(n, d)$, the statements and proofs of all of the results through Section 5 hold with coefficients in any Noetherian commutative ring of finite global dimension.

In Sections 6 and 7 we use the field assumption explicitly. It seems likely that this can be gotten around, for example by using the results of [AHR12].

2.2. The Schur Functor. Using the action of the symmetric group on $E^{\otimes d}$ on the right, one can define a functor from $C_{k}(n, d)$ to the category of representations of the symmetric group $\mathfrak{S}_{d}$.

Definition 2.2. The Schur functor is defined as

$$
\operatorname{Hom}\left(E^{\otimes d},-\right): C_{k}(n, d) \rightarrow k \mathfrak{S}_{d^{-}} \text {Mod. }
$$

From this definition, it is clear that it admits a left adjoint. A slightly different description of the Schur functor also yields a right adjoint (cf. [DEN04]). We interpret the Schur functor geometrically and obtain similar descriptions for its adjoints as well.

2.3. The nilpotent cone for $G L_{d}$. For any commutative $\mathbb{C}$-algebra $R$, the $R$ points of $\mathfrak{g}_{d}$ is the set of endomorphisms of the free $R$-module $R^{d}$. The $R$-points of the quotient stack $\left[\mathfrak{g}_{d} / G_{d}\right]$ is the groupoid whose objects are endomorphisms of locally free $R$-modules of rank $d$ and morphisms are isomorphisms between such pairs.

Let $\mathcal{N}_{d} \subset \mathfrak{g}_{d}$ denote the nilpotent cone, the variety parametrizing nilpotent endomorphisms of $\mathbb{C}^{d}$. For $R$ a commutative $\mathbb{C}$-algebra, the set of $R$-points of $\mathcal{N}_{d}$ is the set of nilpotent endomorphisms of $R^{d}$ and the groupoid of $R$-points of 
$\left[\mathcal{N}_{d} / G_{d}\right]$ consists of nilpotent endomorphisms of locally free $R$-modules of rank $d$ and isomorphisms between them.

We will be interested in the category $P_{G_{d}}\left(\mathcal{N}_{d} ; k\right)$ of equivariant perverse sheaves on the nilpotent cone. The $G_{d}$-orbits in $\mathcal{N}_{d}$ are equivariantly simply connected and labeled by partitions of $d$ according to the Jordan decomposition.

2.4. The Springer and Grothendieck resolutions. We now list some basic facts about the Springer and Grothendieck resolutions of $\mathcal{N}$ and $\mathfrak{g}$ for an arbitrary reductive group. For a more detailed account, see [CG97, Chapter 3].

Recall that if $\mathfrak{h} \subset \mathfrak{g}$ is a Cartan subalgebra, then Chevalley's restriction theorem tells us that $\mathfrak{g} / / G \cong \mathfrak{h} / W$. Let $\chi: \mathfrak{g} \rightarrow \mathfrak{g} / / G$ be the quotient map. Note that $\chi^{-1}(0)=\mathcal{N}$. Let $\mathfrak{h}_{\text {reg }} \subset \mathfrak{h}$ be the complement of the reflection hyperplanes.

Recall the existence of the Grothendieck simultaneous resolution $\pi: \tilde{\mathfrak{g}} \rightarrow \mathfrak{g}$, the Springer resolution $\pi_{\mathcal{N}}: \tilde{\mathcal{N}} \rightarrow \mathcal{N}$ and the map $\tilde{\chi}: \tilde{\mathfrak{g}} \rightarrow \mathfrak{h}$, which fit into the following diagram:

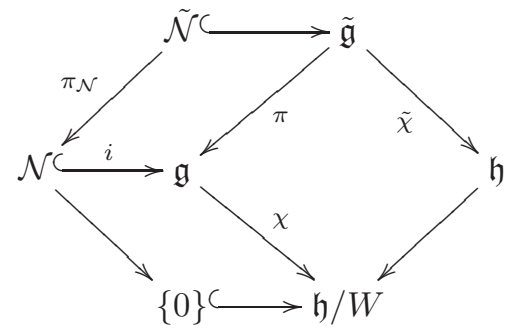

The map $\pi_{\mathcal{N}}$ is semi-small, $\pi$ is small and both are proper. The restriction of the map $\chi$ to $\mathfrak{g}_{\mathrm{rs}}=\chi^{-1}\left(\mathfrak{h}_{\mathrm{reg}} / W\right)$ is smooth with fibers isomorphic to $G / T$. The fundamental group of $\mathfrak{h}_{\text {reg }} / W$ is the braid group $B_{W}$. As the fibers of $\chi$ over $\mathfrak{h}_{\text {reg }} / W$ are simply connected, the fundamental group of $\mathfrak{g}_{\mathrm{rs}}$ is also the braid group. The restriction of $\pi$ to $\tilde{\mathfrak{g}}_{\mathrm{rs}}$ is the $W$-cover corresponding to the subgroup of pure braids $P_{W}$. In particular, the short exact sequence $1 \rightarrow P_{W} \rightarrow B_{W} \rightarrow W \rightarrow 1$ arises from the long exact sequence of homotopy groups for the Galois cover $\tilde{\mathfrak{g}}_{\mathrm{rs}} \rightarrow \mathfrak{g}_{\mathrm{rs}}$.

In the case $G=G_{d}$, the quotient $\mathfrak{h} / W=\mathbb{A}^{d} / \mathfrak{S}_{d}$ is naturally isomorphic to the affine space $Q_{d}$ of monic polynomials of degree $d$. Under this isomorphism, $\chi$ is identified with the map sending an endomorphism to its characteristic polynomial. The regular locus $\mathfrak{h}_{\text {reg }} / W$ is identified with the open subvariety $\left(Q_{d}\right)_{\text {reg }} \subset Q_{d}$ consisting of monic polynomials with $d$ distinct roots.

2.5. Springer theory. Here we review Juteau's study of modular Springer theory [Jut07]. Before doing so, we remark that while Juteau works with varieties over finite fields, we consider the analogous situation over the complex numbers.

Let $\mathcal{S}=\pi_{\mathcal{N} * \underline{k}_{\tilde{\mathcal{N}}}}[\operatorname{dim} \tilde{\mathcal{N}}]$ denote the Springer sheaf and $\mathcal{G}=\pi_{*} \underline{k}_{\tilde{\mathfrak{g}}}[\operatorname{dim} \mathfrak{g}]$ the Grothendieck sheaf.

There exists a Fourier-Sato transform functor, denoted

$$
\mathbb{T}: D_{G}(\mathfrak{g} ; k) \rightarrow D_{G}\left(\mathfrak{g}^{*} ; k\right) .
$$

It is defined by composing the functor defined in [KS94, 33.7$]$ with the shift [dim $\mathfrak{g}]$. With this shift, $\mathbb{T}$ is $t$-exact for the perverse $t$-structure [KS94, Proposition 10.3.18]. This functor is an equivalence of categories with inverse

$$
' \mathbb{T}: D_{G}\left(\mathfrak{g}^{*} ; k\right) \rightarrow D_{G}(\mathfrak{g} ; k) .
$$


We fix an isomorphism $\mathfrak{g}^{*} \cong \mathfrak{g}$ and will identify them from now on. Brylinski [Bry86, §11] proves the following proposition and Juteau [Jut07] observes that it still holds in the modular case.

Proposition 2.3. There is a natural isomorphism $\mathbb{T}(\mathcal{S}) \cong \mathcal{G}$.

Using this, Juteau defines a modular Springer correspondence from irreducible representations of $W$ to IC-sheaves on $\mathcal{N}$. The correspondence associates to an irreducible $W$-representation the image of the functor $\mathbb{T} j_{\mathrm{rs} ! *}$ applied to the corresponding local system on $\mathfrak{g}_{\mathrm{rs}}$. The $\mathbf{I C}$-sheaves that occur in the correspondence are precisely those contained in the top (or equivalently in the socle) of the Springer sheaf. To see that this is true, note that the socle of $k[W]$ consists of the irreducible representations of $W$ with positive multiplicities. Thus the socle of $j_{! *} j^{*} \mathcal{G}=\mathcal{G}$ consists of the !*-extensions of local systems corresponding to irreducible $W$-representations.

The Grothendieck sheaf $\mathcal{G}$ carries a natural $W$-action because it is a GoreskyMacPherson extension of a pushforward along a $W$-cover. Using this action, we can equip the Springer sheaf $\mathcal{S}$ with a $W$-action in two ways: by the Fourier transform or by restriction to the nilpotent cone.

Proposition 2.4. The two $W$-actions on $\mathcal{S}$ - one defined by Fourier transform and the other by restriction to the nilpotent cone - differ by the sign character. In other words, there is an isomorphism of $W$-sheaves:

$$
\mathbb{T}(\mathcal{G}) \cong i^{*} \mathcal{G} \otimes \operatorname{sgn} .
$$

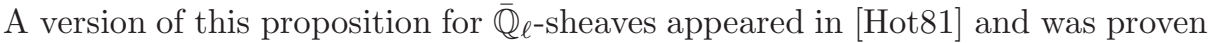
for $\mathcal{D}$-modules in [Gin83] and [HK84]. A proof in the modular case is given in [AHJR]).

2.6. The affine Grassmannian. We first recall the affine Grassmannian for $G L_{n}$, which will play a role analogous to $\mathcal{N}_{d}$, and then the Beilinson-Drinfeld Grassmannian, which corresponds under the same analogy to $\mathfrak{g}_{d}$.

2.6.1. Local version. Let $\mathcal{G} r$ denote the affine Grassmannian over $\mathbb{C}$ for the group $G_{n}=G L_{n}$. In other words, $\mathcal{G} r$ is the ind-scheme over $\mathbb{C}$ whose $R$-points form the set $G_{n}(\mathcal{K}) / G_{n}(\mathcal{O})$ where $\mathcal{K}=R((t))$ is the ring of formal Laurent series and $\mathcal{O}=R[[t]]$ is the ring of formal power series, for $R$ a commutative $\mathbb{C}$-algebra. The group $G_{n}(\mathcal{K})$ acts transitively on the set of $\mathcal{O}$-lattices in $\mathcal{K}^{\oplus n}$, and the stabilizer of the standard lattice is $G_{n}(\mathcal{O})$. It follows that one can view the $R$-points of $\mathcal{G r}$ as lattices.

For each $\lambda \in \Lambda_{n}^{+}$, we associate the orbit $\mathcal{G} r^{\lambda}$ in $\mathcal{G r}$ of the group scheme with $R$-points $G_{n}(\mathcal{O})$. Let $\mathbf{d}=(d, 0, \ldots, 0) \in \Lambda_{n}^{+}$. We will be interested in $\overline{\mathcal{G r}^{\mathbf{d}}}$ and $G_{n}(\mathcal{O})$-perverse sheaves supported on it. Let $\mathcal{O}_{d}$ denote the quotient $\mathcal{O} / t^{d} \mathcal{O}$. As the congruence subgroup $\operatorname{Ker}\left(G_{n}(\mathcal{O}) \rightarrow G_{n}\left(\mathcal{O}_{d}\right)\right)=1+t^{d} \mathfrak{g}_{n}(\mathcal{O})$ acts trivially on $\overline{\mathcal{G r}^{\mathbf{d}}}$, it is equivalent to study $P_{G_{n}\left(\mathcal{O}_{d}\right)}\left(\overline{\mathcal{G r}^{\mathbf{d}}} ; k\right)$. From the definitions above, one finds that $\overline{\mathcal{G} r}$ is a projective variety parametrizing lattices $L$ contained in the standard lattice $L_{0}=\mathcal{O}^{\oplus n}$ such that the quotient $L_{0} / L$ is a locally free $R$-module of rank $d$.

The $G_{n}(\mathcal{O})$-orbits in $\overline{\mathfrak{G r}^{\mathbf{d}}}$ are labeled by the set $\Lambda(n, d) \cap \Lambda_{n}^{+}=\left\{\left(\lambda_{1}, \ldots, \lambda_{n}\right) \in\right.$ $\left.\Lambda_{n} \mid \lambda_{1} \geq \ldots \geq \lambda_{n} \geq 0, \sum \lambda_{i}=d\right\}$. As $n \geq d$, this set is in natural bijection with the set of partitions of $d$. 
Let $\varpi_{1}$ be the fundamental weight $(1,0, \ldots, 0)$. There is a $G(\mathcal{O})$-equivariant semi-small resolution $\left(\mathcal{G} r^{\varpi_{1}}\right)^{* d} \rightarrow \overline{\mathcal{G} r}{ }^{\mathbf{d}}$. The $\mathbb{C}$-points of $\left(\mathcal{G}^{\varpi_{1}}\right)^{* d}$ over a point $L \in \overline{\mathcal{G} r^{\mathbf{d}}}$ are given by all flags $0 \subset V^{1} \subset V^{2} \subset \ldots \subset V^{d-1} \subset L_{0} / L$ preserved by the action of $\mathcal{O}$. Note that $\left(\mathcal{G} r^{\varpi_{1}}\right)^{* d}$ is smooth of dimension $(n-1) d$.

2.6.2. Global version. The $d$-th Beilinson-Drinfeld Grassmannian [BD, MV07a] of $G L_{n}$, denoted by $\mathfrak{G}(n, d)$, is an ind-scheme defined over $Q_{d}$, the space of monic polynomials of degree $d$, whose $R$-points are isomorphism classes of triples $(P, \mathcal{F}, \beta)$ where $P \in Q_{d}(R), \mathcal{F}$ is a rank $n$ vector bundle on $\mathbb{A}^{1}$, and $\beta$ is a trivialization of $\mathcal{F}$ away from the zeros of $P$ in $\mathbb{A}^{1}$.

Let $\mathfrak{K}$ be the ring of rational functions $R(t)$ and $\mathfrak{O}$ the ring of polynomials $R[t]$. Let $\mathcal{L}_{0}$ denote the standard $\mathfrak{O}$-lattice in $\mathfrak{K}^{\oplus n}$.

Following [Ngô99, MV07b], we will be interested in a particular smooth, nddimensional closed subscheme of $\mathfrak{G}(n, d)$ which we denote by $\mathfrak{G}_{d}$. The $R$-points of $\mathfrak{G}_{d}$ are the $\mathfrak{O}$-lattices $\mathcal{L} \subset \mathcal{L}_{0}$ such that $\mathcal{L}_{0} / \mathcal{L}$ is a locally free $R$-module of rank $d$. To see that this is a subfunctor of $\mathfrak{G}(n, d)$, note that any such lattice is a locally free $\mathfrak{O}$-module of rank $n$, i.e., a vector bundle of rank $n$ on $\mathbb{A}^{1}$.

Equivalently, the points of $\mathfrak{G}_{d}$ can be expressed as

$$
\mathfrak{G}_{d}(R)=\left\{g \mathcal{L}_{0} \subset \mathcal{L}_{0} \mid g \in G_{n}(\mathfrak{K}) \cap \mathfrak{g}_{n}(\mathfrak{O}), \operatorname{deg}(\operatorname{det}(g))=d\right\} .
$$

From this point of view, the natural map to $Q_{d}$ is defined by sending a lattice $\mathcal{L}=g \mathcal{L}_{0}$ to the determinant of $g$.

Let $G_{n, d}$ be the group scheme over $Q_{d}$ whose fiber at a point $P \in Q_{d}(R)$ has $R$-points $G_{n, d}(P)(R)=G L_{n}(\mathfrak{D} /(P))$.

Ngô checks in $[\mathrm{Ngô} 99,2.1 .1]$, that $G_{n, d} \rightarrow Q_{d}$ is smooth with geometrically connected fibers of dimension $n^{2} d$. It comes with a natural action $G_{n, d} \times{ }_{Q_{d}} \mathfrak{G}_{d} \rightarrow$ $\mathfrak{G}_{d}$.

We will consider the stack $\left[\mathfrak{G}_{d} / G_{n, d}\right]$. Its $R$-points form the groupoid whose objects are pairs $(L \subset F)$ where $F$ is a locally free $\mathfrak{O}$-module of rank $n$ with $L$ a submodule, such that $F / L$ is locally free over $R$ of rank $d$. Again, $\left[\overline{\mathcal{G r}^{\mathbf{d}}} / G_{n}\left(\mathcal{O}_{d}\right)\right]$ is then simply the subfunctor of such pairs where $P=x^{d}$.

Analogous to the Grothendieck resolution, there is a global resolution, which we denote by $\tilde{\mathfrak{G}}_{d}$. It is the scheme whose $R$-points are full flags of $\mathfrak{O}$-lattices $\mathfrak{D}^{n}=\mathcal{V}^{0} \supset \mathcal{V}^{1} \supset \ldots \supset \mathcal{V}^{d}$ such that $\mathcal{V}^{i} / \mathcal{V}^{i-1}$ is a locally free $R$-module of rank 1 . The map $\pi_{\mathfrak{G}}: \tilde{\mathfrak{G}}_{d} \rightarrow \mathfrak{G}_{d}$ is defined by $\pi_{\mathfrak{G}}\left(\mathcal{V}^{0} \supset \ldots \supset \mathcal{V}^{d}\right)=\mathcal{V}_{d}$

Observe that the fibers of $\mathfrak{G}_{d}, G_{n, d}$, and $\tilde{\mathfrak{G}}_{d}$ over $x^{d} \in Q_{d}$ are respectively $\overline{\mathcal{G r}^{\mathbf{d}}}$, $G_{n}\left(\mathcal{O}_{d}\right)$, and $\left(\mathcal{G r}^{\varpi_{1}}\right)^{* d}$. The spaces described fit into the following diagram analogous to that of Springer theory.

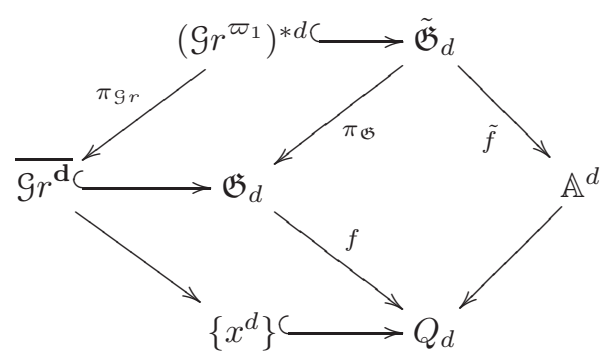


Similarly to above, $\pi_{\mathfrak{G} r}$ is semi-small, $\pi_{\mathfrak{G}}$ is small and both are proper. Let $\left(\mathfrak{G}_{d}\right)_{\mathrm{rs}}=f^{-1}\left(\left(Q_{d}\right)_{\mathrm{reg}}\right),\left(\tilde{\mathfrak{G}}_{d}\right)_{\mathrm{rs}}=\pi_{\mathfrak{G}}^{-1}\left(\left(\mathfrak{G}_{d}\right)_{\mathrm{rs}}\right)$, and $j_{\mathfrak{G} \mathrm{rs}}:\left(\mathfrak{G}_{d}\right)_{\mathrm{rs}} \rightarrow \mathfrak{G}_{d}$ be the inclusion. The restriction $\pi_{\mathfrak{G}, \mathrm{rs}}:\left(\tilde{\mathfrak{G}}_{d}\right)_{\mathrm{rs}} \rightarrow\left(\mathfrak{G}_{d}\right)_{\mathrm{rs}}$ of $\pi_{\mathfrak{G}}$ is a Galois $\mathfrak{S}_{d}$-cover. It follows that

$$
\left(\pi_{\mathfrak{G}}\right)_{*}{\underline{\tilde{G}_{d}}}_{\tilde{\mathfrak{B}}_{d}}[n d] \cong\left(j_{\mathfrak{G r s}}\right) ! *\left(\pi_{\mathfrak{G}, \mathrm{rs}}\right)_{*} \underline{k}_{\left(\tilde{\mathfrak{G}}_{d}\right)_{\mathrm{rs}}}[n d] .
$$

2.7. Geometric Satake. Mirković and Vilonen [MV07a] prove, extending work in characteristic zero of Lusztig [Lus83], Beilinson-Drinfeld [BD] and Ginzburg [Gin95], that for a split reductive group $G$ defined over a Noetherian commutative ring k of finite global dimension: the category $P_{G^{\vee}(\mathcal{O})}(\mathcal{G} r ; \mathbf{k})$ of equivariant perverse sheaves with coefficients in $\mathbf{k}$ on the affine Grassmannian for the Langlands dual group $G^{\vee} / \mathbb{C}$, together with a natural convolution product $*$, is tensor equivalent to the category of representations of $G$ over k.

In this paper, we only consider the case $G=G^{\vee}=G L_{n}$ and $\mathbf{k}=k$ is a field. We use the following immediate corollaries of the results of [MV07a].

Theorem 2.5. (1) There is an equivalence of categories

$$
P_{G_{n}(\mathcal{O})}\left(\overline{\mathcal{G r}}{ }^{\mathbf{d}} ; k\right) \cong \mathcal{C}_{k}(n, d) .
$$

(2) Under this equivalence, the $G L_{n}$-representation $E^{\otimes d}$ (here $E$ is the standard $n$-dimensional representation) corresponds to the $d$-fold convolution product

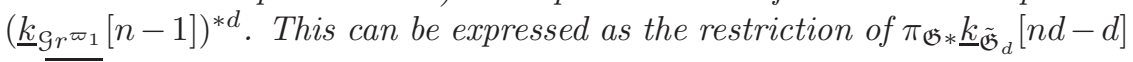
to $\overline{\mathcal{G r}^{\mathbf{d}}}$, or equivalently as the pushforward $\pi_{\mathcal{G} r * k_{\left(\mathcal{G} \varpi_{1}\right) * d}}[n d-d]$.

(3) The symmetric group $\mathfrak{S}_{d}$ acts on

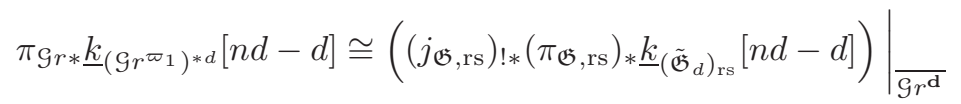

by the deck transformations of $\pi_{\mathfrak{G}, \mathrm{rs}}$ and the funtoriality of !*-extensions. This action corresponds under the geometric Satake equivalence to the permutation action of $\mathfrak{S}_{d}$ on $E^{\otimes d}$.

\section{A projeCtion map AND Lusztig's SECTION}

In this section, we relate the spaces $\mathcal{N}_{d}$ and $\overline{\mathcal{G r}^{\mathbf{d}}}$ and their quotient stacks. We do so using the functor of points descriptions given in the previous sections.

Lemma 3.1. There exist natural morphisms:
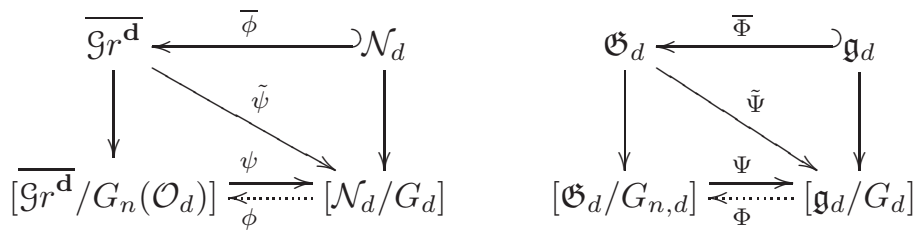

such that all of the solid morphisms form a commutative diagram, while the dotted morphisms satisfy $\psi \circ \phi=\operatorname{Id}_{\left[\mathcal{N}_{d} / G_{d}\right]}$ and $\Psi \circ \Phi=\operatorname{Id}_{\left[\mathfrak{g}_{d} / G_{d}\right]}$.

Proof. Recall diagrams 2.1 and 2.2. In the lemma above, the morphisms denoted by lower-case letters will be defined as the restriction of the upper-case morphisms by the closed embeddings $\mathcal{N}_{d} \rightarrow \mathfrak{g}_{d}$ and $\overline{\mathcal{G r}^{\mathbf{d}}} \rightarrow \mathfrak{G}_{d}$. We thus need only describe the global (or upper-case) morphisms and check that they satisfy the relations described. 
The maps $\tilde{\Psi}$ and $\Psi$. The map $\tilde{\Psi}$ is the forgetful map which associates to an $R$-point $\mathcal{L}$ of $\mathfrak{G}_{d}$ the rank $d$ locally free $R$-module $\mathcal{L}_{0} / \mathcal{L}$ together with the endomorphism given by the action of $t \in \mathfrak{O}$. Similarly, $\Psi$ associates to an $R$-point $(P, L \subset F)$ of $\left[\mathfrak{G}_{d} / G_{n, d}\right]$, the locally free $R$-module $F / L$ together with the endomorphism defined by multiplication by $t$.

The maps $\bar{\Phi}$ and $\Phi$. For each $R$-point $(a, E)$ in $\left[\mathfrak{g}_{d} / G_{d}\right]$, where $a$ is an endomorphism of a rank $d$ locally free $R$-module $E$, let $\Phi(a) \in\left[\mathfrak{G}_{d} / G_{n, d}\right]$ be the pair $(L \subset F)$, where $F:=E[t] \oplus \mathfrak{O}^{\oplus n-d}$ and $L:=(a-t) E[t] \oplus \mathfrak{O}^{\oplus n-d}$. To see that this is indeed a point of $\left[\mathfrak{G}_{d} / G_{n, d}\right]$, note that $a-t \in G L(E(t)) \cap \mathfrak{g l}(E[t])$ and has determinant equal to the characteristic polynomial of $a$ which has degree $d$ as desired. The map $\bar{\Phi}$ is defined analogously.

It remains to exhibit a natural equivalence $\mathrm{Id} \stackrel{\sim}{\rightarrow} \Psi \circ \Phi$.

We claim that such an equivalence can be defined as follows. For any $(a, E)$, consider the map of $R$-modules given by

$$
E \hookrightarrow E[t] \rightarrow E[t] /(a-t) E[t] .
$$

Note that the map is injective as $E \cap(a-t) E[t]=0$. To prove surjectivity, one can use induction on the degree to show that any polynomial $p(t) \in E[t]$ is in $E+(a-t) E[t]$. To finish the proof, we observe that this isomorphism intertwines the action of $a$ on $E$ with the action of $t$ on $E[t] /(a-t) E[t]$.

Remark 3.2. The local version $\bar{\phi}$ was first observed by Lusztig [Lus81]. As far as I am aware, a description of the global map $\bar{\Phi}$ first appeared in [MV07b]. The maps in the other direction were known to some experts, but it seems the relationship to Lusztig's map had not been previously noticed.

Remark 3.3. Lusztig [Lus81] observes that the map $\bar{\phi}$ is an open embedding when $n=d$ and the same is true of $\bar{\Phi}$. Moreover, the image of the embedding intersects every orbit and provides a natural bijection between the orbits of $\overline{\mathcal{G} r^{\mathbf{d}}}$ and those of $\mathcal{N}_{d}$

\section{Equivalence of Categories}

In this section we use the Lemma 3.1 from the previous section to prove equivalences of categories, which we then use to deduce Theorem 1.1.

Theorem 4.1. The maps $\phi: \mathcal{N}_{d} \rightarrow \overline{\mathcal{G r}^{\mathbf{d}}}$ and $\Phi: \mathfrak{g}_{d} \rightarrow \mathfrak{G}_{d}$ induce equivalences of categories

$$
\begin{aligned}
\phi^{\bullet} & :=\phi^{*}\left[d^{2}-n d\right]: P_{G_{n}(\mathcal{O})}\left(\overline{\mathcal{G r}^{\mathbf{d}}}\right) \stackrel{\sim}{\rightarrow} P_{G_{d}}\left(\mathcal{N}_{d}\right), \\
\Phi^{\bullet} & :=\Phi^{*}\left[d^{2}-n d\right]: P_{G_{n, d}}\left(\mathfrak{G}_{d}\right) \stackrel{\sim}{\rightarrow} P_{G_{d}}\left(\mathfrak{g}_{d}\right) .
\end{aligned}
$$

Proof. By [BBD82, 4.2.5], the (shifted) pull-back along a smooth morphism $f$ : $X \rightarrow Y$ with connected fibers is a fully faithful embedding of the category of perverse sheaves on $Y$ to that on $X$.

We now check that $\Phi$ enjoys these properties (and therefore $\phi$ does as well by base change). From this it will follow that $\Phi^{\bullet}$ and $\phi^{\bullet}$ are fully faithful.

First consider the case $n=d$. Recall that $\bar{\phi}$ and $\bar{\Phi}$ are open morphisms (Remark 3.3). From this, we can deduce that $\phi$ and $\Phi$ are smooth. 
By Lemma 3.1, $\Psi \circ \Phi=\operatorname{Id}_{\left[\mathfrak{g}_{d} / G_{d}\right]}$ and thus the fibers of $\Phi$ are connected as long as they are nonempty. To see that the fibers are non-empty, note that every $G_{n, d}$-orbit of $\mathfrak{G}_{d}$ has non-trivial intersection with $\mathfrak{g}_{d}$.

In the general case $n>d$, we can factor $\tilde{\Phi}_{d}^{n}$ as $\tilde{\Phi}_{d}^{d}$ and the map $a_{d, n}:\left[\mathfrak{G}_{d} / G_{d, d}\right] \rightarrow$ $\left[\mathfrak{G}_{n} / G_{n, d}\right]$. In [Ngô99, Lemme 2.2.1], it is shown that $a_{d, n}$ is smooth with connected fibers.

By Lemma 3.1, the compositions $\phi^{*} \circ \psi^{*}$ and $\Phi^{*} \circ \Psi^{*}$ are the identity functors on $P_{G_{d}}\left(\mathcal{N}_{d}\right)$ and $P_{G_{n, d}}\left(\mathfrak{G}_{d}\right)$ respectively. We conclude that $\phi^{\bullet}$ and $\Phi^{\bullet}$ are also essentially surjective, which completes the proof.

We can now prove Theorem 1.1:

Proof. Composing the equivalence from Theorem 2.5 and the first equivalence from Theorem 4.1 give the desired result.

We conclude this section with an observation that we will not use in what follows, but which puts in perspective the relationship between the various equivariant derived categories.

Corollary 4.2. The pullback functor on equivariant derived categories

$$
\psi^{*}: D_{G_{d}}\left(\mathcal{N}_{d}\right) \rightarrow D_{G_{n}(\mathcal{O})}\left(\overline{\mathcal{G r}^{\mathbf{d}}}\right)
$$

(resp. $\Psi^{*}: D_{G_{d}}\left(\mathfrak{g}_{d}\right) \rightarrow D_{G_{n, d}}\left(\mathfrak{G}_{d}\right)$ ) splits the functor

$$
\phi^{*}: D_{G_{n}(\mathcal{O})}\left(\overline{\mathcal{G r}}{ }^{\mathbf{d}}\right) \rightarrow D_{G_{d}}\left(\mathcal{N}_{d}\right)
$$

(resp. $\left.\Phi^{*}: D_{G_{n, d}}\left(\mathfrak{G}_{d}\right) \rightarrow D_{G_{d}}\left(\mathfrak{g}_{d}\right)\right)$, in the following sense:

For any two objects, $A, B \in D_{G_{d}}\left(\mathcal{N}_{d}\right), \psi^{*}$ induces an injection of graded vector spaces

$$
\operatorname{Ext}_{D_{G_{d}}\left(\mathcal{N}_{d}\right)}^{*}(A, B) \hookrightarrow \operatorname{Ext}_{D_{G_{n}(\mathcal{O})}^{*}\left(\overline{\mathcal{G r}^{\mathbf{d}}}\right)}\left(\psi^{*} A, \psi^{*} B\right),
$$

which naturally splits the projection map given by $\phi^{*}$.

Remark 4.3. It is worth noting that as functors between equivariant derived categories, the pullback functors do not induce equivalences, and in fact the various categories are not equivalent. To see this, consider the Ext-groups between the !and $*$-extension of the constant sheaf on the stratum associated to a partition $\lambda$. The Ext groups in the equivariant derived categories will agree with the equivariant cohomology of the stratum, which in turn agrees with the group cohomology (of the reductive part) of the stabilizer of a point. Unless $n=d$ and $\lambda$ is the trivial partition, these will not agree.

\section{Generalized Schur-Weyl Theorem}

In this section we prove Theorem 1.2.

Proof. Recall the following consequences of the geometric Satake equivalence summarized in Theorem 2.5. There is natural isomorphism

$$
\operatorname{End}_{G_{n}}\left(E^{\otimes d}\right) \cong \operatorname{End}_{P_{G_{n}(\mathcal{O})}(\mathcal{G} r)}\left(\pi_{\left.\mathcal{G} r * \underline{k}_{\left(\mathcal{G} \varpi_{1}\right)^{* d}}[n d-d]\right)}\right. \text {. }
$$

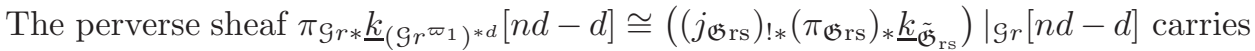
an action of $\mathfrak{S}_{d}$ induced by the action of the deck-transformations of $\pi_{\mathfrak{G r s}}$, and this action agrees under the isomorphism above with the permutation action of $\mathfrak{S}_{d}$ on $E^{\otimes d}$. 
We wish to translate this action from the affine Grassmannian to the nilpotent cone. A simple generalization of the maps $\bar{\Phi}$ and $\bar{\phi}$ completes the following commutative cube:

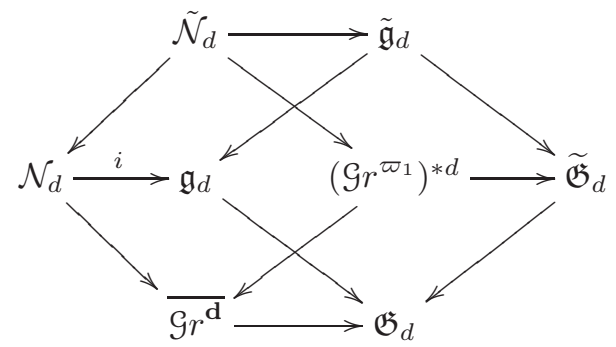

Here the square involving $\mathcal{N}$ 's and $\mathcal{G} r$ 's and the square involving $\mathfrak{g}$ 's and $\mathfrak{G}$ 's are both pull-back squares, all the maps from upper-right to lower-left are proper, and the maps from upper-left to lower-right are inclusions.

By Theorem 4.1, the functor $\phi^{\bullet}$ induces an isomorphism

$$
\operatorname{End}_{P_{G_{n}(\mathcal{O})}(\mathcal{G} r)}\left(\pi_{\left.\mathcal{G} r * \underline{k}_{(\mathcal{G}} r^{\varpi_{1}}\right)_{* d}}[n d-d]\right) \cong \operatorname{End}_{P_{G_{d}}\left(\mathcal{N}_{d}\right)}(\mathcal{S}) .
$$

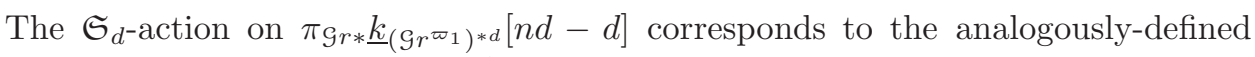
action on $\mathcal{S} \cong i^{*}\left(j_{\mathrm{rs}}\right) ! * \pi_{\mathrm{rs} * \underline{\mathfrak{g}}_{\mathrm{rs}}}\left[d^{2}-d\right]$.

On the other hand, Juteau [Jut07] observes that, as in characteristic 0, the Fourier transform exchanges the Springer and Grothendieck sheaves and so

$$
\operatorname{End}(\mathcal{S}) \stackrel{\mathbb{T}}{\cong} \operatorname{End}(\mathcal{G}) \cong \operatorname{End}\left(\pi_{\mathrm{rs} *} \underline{\underline{\mathfrak{g}}}_{\mathrm{rs}}\left[d^{2}\right]\right) \cong k \mathfrak{S}_{d}
$$

Proposition 2.4 says that the resulting action of $\mathfrak{S}_{d}$ by Fourier transform differs from the one arising by restriction (as in the previous paragraph) by a sign character. But as the first induces an isomorphism, the second does as well.

\section{Geometric Schur Functor}

The previous section allows us to identify the functor $\operatorname{Hom}(\mathcal{S},-)$ with the Schur functor. In other words, we have a commutative diagram of functors:

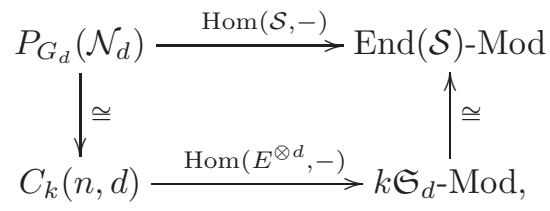

where the action of $\mathfrak{S}_{d}$ on $\mathcal{S}$ is through the action by deck transformations on $\mathcal{G}$ and the identification of $\mathcal{S}$ with $i^{*} \mathcal{G}[-d]$.

Motivated by this connection, we will shift our focus to a general connected complex reductive group $G$. By analogy, we can define a Schur functor from the category of $G$-equivariant perverse sheaves on the nilpotent cone in $\mathfrak{g}$ to representations of the Weyl group,

$$
\operatorname{Hom}(\mathcal{S},-): P_{G}\left(\mathcal{N}_{G} ; k\right) \rightarrow \operatorname{End}(\mathcal{S}) \text {-Mod. }
$$

In this section, we will introduce another functor that is closely related to $\operatorname{Hom}(\mathcal{S},-)$. We propose that this new functor should be viewed as a geometric Schur functor for $G$. 
To ease notation, we will let $j=j_{\mathrm{rs}}$. We consider the functor:

$$
\mathcal{F}:=j^{*} \circ \mathbb{T} \circ i_{*}: P_{G}(\mathcal{N}) \rightarrow P_{G}\left(\mathfrak{g}_{\mathrm{rs}}\right) .
$$

Note that it is exact because each component is exact. Let $\operatorname{Loc}_{W}\left(\mathfrak{g}_{\mathrm{rs}}\right) \subset P_{G}\left(\mathfrak{g}_{\mathrm{rs}}\right)$ denote the full subcategory of local systems with monodromy factoring through the Weyl group shifted to be perverse.

In the proof of the following lemma we will use parabolic restriction and induction functors. For their definition and some basic properties in this context, see for example [AM12].

Lemma 6.1. The functor $\mathcal{F}$ factors through the inclusion $\operatorname{Loc}_{W}\left(\mathfrak{g}_{\mathrm{rs}}\right) \subset P_{G}\left(\mathfrak{g}_{\mathrm{rs}}\right)$.

Proof. We first show that the statement is true for all simple objects in $P_{G}(\mathcal{N} ; k)$. For each nilpotent orbit $\mathcal{O}$ and irreducible local system $\mathcal{L} \in P_{G}(\mathcal{O} ; k)$, consider the IC-sheaf $A=\mathbf{I C}(\mathcal{O}, \mathcal{L})$. Let $T$ be a maximal torus for $G$. Note that $\operatorname{res}_{T}^{G} A \neq 0$ if and only if $A$ is in Juteau's modular Springer correspondence and then $\mathbb{T} A \cong$ $\operatorname{IC}\left(\mathfrak{g}_{\mathrm{rs}}, \mathcal{L}_{V}\right)$, where $\mathcal{L}_{V} \in \operatorname{Loc}_{W}\left(\mathfrak{g}_{\mathrm{rs}}\right)$ and corresponds to some irreducible $k[W]$ representation $V$.

Suppose instead that $\operatorname{res}_{T}^{G} A=0$. Then there exists some Levi subgroup $L$ such that $A_{0}=\operatorname{res}_{L}^{G} A$ is non-zero but parabolic restriction of $A_{0}$ for any proper Levi subgroup of $L$ vanishes. It follows that the same is true of $\mathbb{T} A_{0}$ and by [Mir04, Lemma 4.4 $]^{1}$, that $\mathbb{T} A_{0}$ is supported on $\mathcal{N}_{L} \times Z(\mathfrak{l})$. By adjunction, there exists a non-zero map $A \rightarrow \operatorname{ind}_{L}^{G} A_{0}$. As $A$ is simple,

$$
\overline{\operatorname{supp} \mathbb{T} A} \subset \overline{\operatorname{ind}_{L}^{G} \mathbb{T} A_{0}} \subset{ }^{G}\left(\mathcal{N}_{L} \times Z(\mathfrak{l})\right) .
$$

Finally, ${ }^{G}\left(\mathcal{N}_{L} \times Z(\mathfrak{l})\right) \cap \mathfrak{g}_{\mathrm{rs}}=\emptyset$ as $L$ is not a maximal torus. Thus $j^{*} \mathbb{T} A=0$.

As $\mathcal{F}$ is exact, we conclude that $\mathcal{F}(X)$ is a local system on $\mathfrak{g}_{\mathrm{rs}}$ for any $X \in P_{G}(\mathcal{N})$. It remains to check that $\mathcal{F} X$ has monodromy that factors through $W$.

Because we have assumed that $k$ is a field, the category of perverse sheaves is Artinian. Thus there exists a minimal $Q \subset X$ such that $\mathcal{F}(X / Q)=0$. By the exactness of $\mathcal{F}, \mathcal{F}(Q) \cong \mathcal{F}(X)$. By the minimality of $Q$, the top of $Q$ is a direct sum of IC-sheaves in Juteau's modular Springer correspondence. Recall that the Springer sheaf is a direct sum of the projective covers of such IC-sheaves. Thus for some $m \geq 0$, there exists a surjection $\mathcal{S}^{\oplus m} \rightarrow Q$. Using again the exactness of $\mathcal{F}$, we conclude that $\mathcal{F}(Q)$ is a quotient of $m$ copies of $j^{*} \mathcal{G}$ and thus has monodromy that factors through the Weyl group.

In order to compare $\mathcal{F}$ with $\operatorname{Hom}(\mathcal{S},-)$, we consider the functor

$$
\rho: \operatorname{End}\left(j^{*} \mathcal{G}\right)-\operatorname{Mod} \rightarrow \operatorname{Loc}_{W}\left(\mathfrak{g}_{\mathrm{rs}}\right)
$$

defined by the tensor product $(-) \otimes_{\operatorname{End}\left(j^{*} \mathcal{G}\right)} j^{*} \mathcal{G}$ and its inverse

$$
\operatorname{Hom}\left(j^{*} \mathcal{G},-\right): \operatorname{Loc}_{W}\left(\mathfrak{g}_{\mathrm{rs}}\right) \rightarrow \operatorname{End}\left(j^{*} \mathcal{G}\right)-\operatorname{Mod}
$$

Theorem 6.2. There is a natural equivalence of functors:

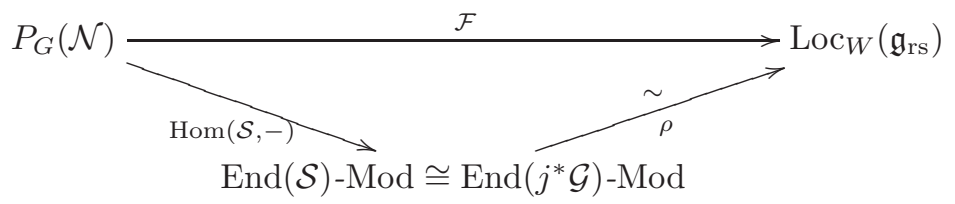

\footnotetext{
${ }^{1}$ While Mirković works with $\mathcal{D}$-modules, the same argument works in the constructible context with arbitrary coefficients.
} 
Proof. We proceed by constructing a sequence of equivalences. Fourier transform induces a equivalence of functors:

$$
\rho(\operatorname{Hom}(\mathcal{S},-)) \cong \rho\left(\operatorname{Hom}\left(\mathcal{G}, \mathbb{T} i_{*}(-)\right)\right) .
$$

For any $X \in P_{G}(\mathcal{N})$, the Fourier transform $\mathbb{T} X$ is perverse and so we obtain an exact sequence:

$$
0 \rightarrow K \rightarrow \mathbb{T} X \rightarrow{ }^{p} j_{*} j^{*} \mathbb{T} X \rightarrow C \rightarrow 0
$$

where $K$ and $C$ have support in $\mathfrak{g}-\mathfrak{g}_{\mathrm{rs}}$. Applying the exact functor $\operatorname{Hom}(\mathcal{G},-)$ gives an isomorphism $\operatorname{Hom}(\mathcal{G}, \mathbb{T} X) \cong \operatorname{Hom}\left(\mathcal{G},{ }^{p} j_{*} j^{*} \mathbb{T} X\right)$. Using the adjunction between $j^{*}$ and ${ }^{p} j_{*}$ we obtain an equivalence:

$$
\rho\left(\operatorname{Hom}\left(\mathcal{G}, \mathbb{T} i_{*}(-)\right)\right) \cong \rho\left(\operatorname{Hom}\left(j^{*} \mathcal{G}, j^{*} \mathbb{T} i_{*}(-)\right)\right) .
$$

The composition $\rho \circ \operatorname{Hom}\left(j^{*} \mathcal{G},-\right)$ restricted to $\operatorname{Loc}_{W}\left(\mathfrak{g}_{\mathrm{rs}}\right)$ is isomorphic to the identity functor. From Lemma 6.1 , the functor $j^{*} \mathbb{T} i_{*}$ takes values in $\operatorname{Loc}_{W}\left(\mathfrak{g}_{\mathrm{rs}}\right)$ and so we may conclude:

$$
\rho\left(\operatorname{Hom}\left(j^{*} \mathcal{G}, j^{*} \mathbb{T} i_{*}(-)\right)\right) \cong j^{*} \mathbb{T} i_{*}(-)=\mathcal{F}(-) .
$$

\section{Geometric Adjoint Functors}

In the previous section we introduced the notion of a geometric Schur functor for an arbitrary connected complex reductive group. We close with a simple observation in this general context.

By the usual adjointness for closed and open embeddings, the geometric Schur functor has the following left and right adjoints which we consider restricted to $\operatorname{Loc}_{W}\left(\mathfrak{g}_{\mathrm{rs}}\right)$ :

$$
\begin{aligned}
& \mathcal{G}_{R}={ }^{p} i^{!} \circ{ }^{'} \mathbb{T} \circ{ }^{p} j_{*}: \operatorname{Loc}_{W}\left(\mathfrak{g}_{\mathrm{rs}}\right) \rightarrow P_{G}(\mathcal{N}), \\
& \mathcal{G}_{L}={ }^{p} i^{*} \circ{ }^{\prime} \mathbb{T} \circ{ }^{p} j_{!}: \operatorname{Loc}_{W}\left(\mathfrak{g}_{\mathrm{rs}}\right) \rightarrow P_{G}(\mathcal{N}) .
\end{aligned}
$$

Lemma 7.1. The functor ${ }^{\prime} \mathbb{T} j_{! *}: \operatorname{Loc}_{W}\left(\mathfrak{g}_{\mathrm{rs}}\right) \rightarrow P_{G}(\mathfrak{g})$ factors through the subcate$\operatorname{gory} P_{G}(\mathcal{N}) \subset P_{G}(\mathfrak{g})$.

In other words, the perverse extension of a local system associated to a representation of the Weyl group is anti-orbital.

Proof. Since $P_{G}(\mathcal{N})$ is a thick subcategory of $P_{G}(\mathfrak{g})$ and $j_{! *}$ preserves epimorphisms, it suffices to check on a projective generator of $\operatorname{Loc}_{W}\left(\mathfrak{g}_{\mathrm{rs}}\right)$. The local system $j^{*} \mathcal{G}$ is such a projective generator and $j_{! *} j^{*} \mathcal{G} \cong \mathcal{G}$. Thus ${ }^{\prime} \mathbb{T} j_{! *} j^{*} \mathcal{G} \cong i_{*} \mathcal{S}$, which has support on $\mathcal{N}$.

Proposition 7.2. The adjoint functors $\mathcal{G}_{L}, \mathcal{G}_{R}$ are both right inverses to $\mathcal{F}$.

Proof. We provide the proof for $\mathcal{G}_{R}$, the proof for $\mathcal{G}_{L}$ is completely analogous.

We seek a natural equivalence $j^{*} \mathbb{T} i_{*}{ }^{p} i^{!} \mathbb{T}^{p} j_{*} \cong \mathrm{Id}$. Consider an element $\mathcal{L} \in$ $\operatorname{Loc}_{W}\left(\mathfrak{g}_{\mathrm{rs}}\right)$. One has the canonical inclusion $j_{!_{*}} \mathcal{L} \hookrightarrow{ }^{p} j_{*} \mathcal{L}$. The Fourier transform $' \mathbb{T}$ is an equivalence of categories, so ${ }^{\prime} \mathbb{T} j_{!_{*}} \mathcal{L} \hookrightarrow{ }^{\prime} \mathbb{T}^{p} j_{*} \mathcal{L}$. By lemma 7.1 , the ${ }^{\prime} \mathbb{T} j_{!_{*}} \mathcal{L}$ is supported on the nilpotent cone $\mathcal{N}$. On the other hand, for any $\mathcal{F} \in P(\mathfrak{g}), i_{*}{ }^{p} i^{!} \mathcal{F}$ is the largest subobject of $\mathcal{F}$ with support in $\mathcal{N}$.

In particular, letting $\mathcal{F}={ }^{1} \mathbb{T}\left({ }^{p} j_{*} \mathcal{L}\right)$ we obtain a commutative triangle: 


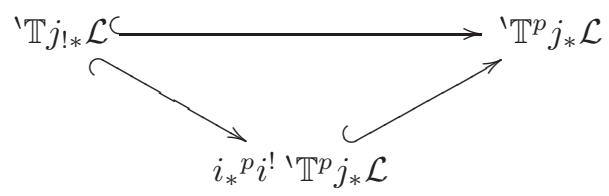

We now apply the exact functor $j^{*} \circ \mathbb{T}$ to obtain:

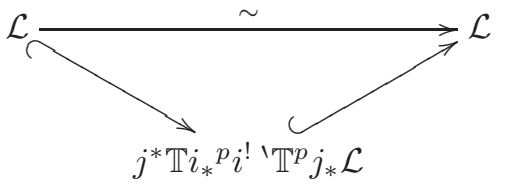

We conclude that the two bottom maps are also isomorphisms and thus $j^{*} \circ \mathbb{T}$ applied to the adjunction morphism gives the natural equivalence we sought to prove.

\section{REFERENCES}

[AH12] P. Achar and A. Henderson. Geometric Satake, Springer correspondence, and small representations. Preprint arXiv:1108.4999, 2012.

[AHJR] P. Achar, A. Henderson, D. Juteau, and S. Riche. Weyl group actions on the Springer sheaf. Proc. London Math. Soc. first published online December 11, 2013 doi:10.1112/plms/pdt055.

[AHJR13] P. Achar, A. Henderson, D. Juteau, and S. Riche. Modular generalized Springer correspondence I: the general linear group. Preprint arXiv:1307.2702, 2013.

[AHR12] P. Achar, A. Henderson, and S. Riche. Geometric Satake, Springer correspondence, and small representations II. Preprint arXiv:1205.5089, 2012.

[AM12] P. Achar and C. Mautner. Sheaves on nilpotent cones, Fourier transform, and a geometric Ringel duality. Preprint arXiv:1207.7044, 2012.

[BBD82] A. Beillinson, J. Bernstein, and P. Deligne. Faisceaux pervers. In Analyse et topologie sur les espaces singuliers, I (Luminy, 1981), volume 100 of Astérisque, pages 5-171. Soc. Math. France, Paris, 1982.

[BD] A. Berlinson and V. Drinfel'd. Quantization of Hitchin integrable system and Hecke eigensheaves. Preprint available at http://www.math.uchicago.edu/ mitya/ langlands/hitchin/BD-hitchin.pdf.

[Bry86] J.-L. Brylinski. Transformations canoniques, dualité projective, théorie de Lefschetz, transformations de Fourier et sommes trigonométriques. Astérisque, 140-141:3-134, 1986. Géométrie et analyse microlocales.

[CG97] N. Chriss and V. Ginzburg. Representation theory and complex geometry. Birkhäuser Boston Inc., Boston, MA, 1997.

[CL74] R. W. Carter and G. Lusztig. On the modular representations of the general linear and symmetric groups. Math. Z., 136:193-242, 1974.

[DEN04] S. R. Doty, K. Erdmann, and D. K. Nakano. Extensions of modules over Schur algebras, symmetric groups and Hecke algebras. Algebr. Represent. Theory, 7(1):67-100, 2004.

[Don87] S. Donkin. On Schur algebras and related algebras. II. J. Algebra, 111(2):354-364, 1987.

[Don93] S. Donkin. On tilting modules for algebraic groups. Math. Z., 212(1):39-60, 1993.

[Gin83] V. Ginsburg. Intégrales sur les orbites nilpotentes et représentations des groupes de Weyl. C. R. Acad. Sci. Paris Sér. I Math., 296(5):249-252, 1983.

[Gin95] V. Ginzburg. Perverse sheaves on a loop group and Langlands duality. Preprint arXiv:alg-geom/9511007, 1995.

[Gre80] J. A. Green. Polynomial representations of $\mathrm{GL}_{n}$, volume 830 of Lecture Notes in Mathematics. Springer-Verlag, Berlin, 1980.

[HK84] R. Hotta and M. Kashiwara. The invariant holonomic system on a semisimple Lie algebra. Invent. Math., 75(2):327-358, 1984. 
[Hot81] R. Hotta. On Springer's representations. J. Fac. Sci. Univ. Tokyo Sect. IA Math., 28(3):863-876 (1982), 1981.

[Jan03] J. C. Jantzen. Representations of algebraic groups, volume 107 of Mathematical Surveys and Monographs. American Mathematical Society, Providence, RI, second edition, 2003.

[Jut07] D. Juteau. Modular Springer correspondence and decomposition matrices. PhD thesis, Université Paris 7 Denis Diderot, 2007. Available at arXiv:0901.3671.

[Kle95] A. S. Kleshchev. Branching rules for modular representations of symmetric groups. I. J. Algebra, 178(2):493-511, 1995.

[KS94] M. Kashiwara and P. Schapira. Sheaves on manifolds, volume 292 of Grundlehren der Mathematischen Wissenschaften [Fundamental Principles of Mathematical Sciences]. Springer-Verlag, Berlin, 1994.

[LO09] Y. Laszlo and M. Olsson. Perverse t-structure on Artin stacks. Math. Z., 261(4):737$748,2009$.

[Lus81] G. Lusztig. Green polynomials and singularities of unipotent classes. Adv. Math., 42:169-178, 1981.

[Lus83] G. Lusztig. Singularities, character formulas, and a $q$-analog of weight multiplicities. In Analyse et topologie sur les espaces singuliers, II, III (Luminy, 1981), volume 101-102 of Astérisque, pages 208-229. Soc. Math. France, Paris, 1983.

[Mar93] S. Martin. Schur algebras and representation theory, volume 112 of Cambridge Tracts in Mathematics. Cambridge University Press, Cambridge, 1993.

[Mau10] C. Mautner. Sheaf Theoretic Methods in Modular Representation Theory. PhD thesis, University of Texas at Austin, 2010.

[Mir04] I. Mirković. Character sheaves on reductive Lie algebras. Mosc. Math. J., 4(4):897-910, 981, 2004.

[MV07a] I. Mirković and K. Vilonen. Geometric Langlands duality and representations of algebraic groups over commutative rings. Ann. of Math. (2), 166(1):95-143, 2007.

[MV07b] I. Mirković and M. Vybornov. Quiver varieties and Beilinson-Drinfeld Grassmannians of type A. Preprint arXiv:0712.4160, 2007.

[Ngô99] B. C. Ngô. Faisceaux pervers, homomorphisme de changement de base et lemme fondamental de Jacquet et Ye. Ann. Sci. École Norm. Sup. (4), 32(5):619-679, 1999. 